\title{
An Architecture for Synchronous Micro-Volunteering in Africa using Social Media
}

\author{
Laurie Butgereit \\ Nelson Mandela Metropolitan University and \\ Meraka Institute, CSIR \\ South Africa \\ lbutgereit@meraka.org.za
}

\author{
Reinhardt A Botha \\ Nelson Mandela Metropolitan University \\ Port Elizabeth, South Afirca \\ ReinhardtA.Botha@nmmu.ac.za
}

\begin{abstract}
Micro-Volunteering is the phenomenon of volunteering one's time and energy for extremely short periods of time. It is often considered a subset of Virtual Volunteering where one can volunteer one's time and energy via the Internet. There are many successful examples of Virtual Volunteering such as the Gutenberg project and the Librivox project. However, in both these cases, a volunteer must donate a substantial amount of his or her time in activities such as reading books or proofreading books. This paper examines the concept of synchronous micro-volunteering where potential volunteers can effectively donate as little as $\mathbf{1 0}$ minutes of their time and immediately assist other people. The paper then describes the architecture which supports synchronous microvolunteering.
\end{abstract}

Keywords-component; micro-volunteering, dr math, mxit

\section{INTRODUCTION}

Many people give their time freely for the benefit of others as a volunteer [1]. People do this for many different reasons. There may be family reasons such as parents volunteering at their children's schools. There may be religious reasons such as people volunteering at a soup kitchen sponsored by their church. There may be civic reasons such as people volunteering at their local police stations or fire stations. There may be recreational reasons such as people volunteering to help at air shows, race tracks, and camp grounds.

Over the past few years, however, the idea of virtual volunteering has grown. These virtual volunteers donate their time and energy via the Internet [2]. There may be many reasons why people prefer to be a virtual volunteer instead of a physical volunteer. The potential volunteer may have employment or family responsibility time constraints and may not have time to visit a charitable organization. The potential volunteer may have a physical disability and may not be physically able to travel to the required location. The potential volunteer may be fearful of traveling to the required location such as a high crime area or an area where there is a medical epidemic.

In such cases virtual volunteering gives these potential volunteers an opportunity to give of their time and energy via the Internet while overcoming their time constraints, physical constraints, and even emotional constraints.
A couple of very successful virtual volunteering projects are discussed in this paper; however, these virtual volunteering projects require a substantial amount of time. This paper describes work towards a synchronous virtual micro-volunteering platform where volunteers can donate as little as ten minutes of their time from their busy days and immediately help others.

\section{EDUCATIONAL LANDSCAPE IN SOUTH AFRICA}

In January 2000, at the start of the new school year, just over one million children (1 080035 to be exact) entered Grade 1 in South Africa [3]. Twelve years later, at the end of Grade 12, only 623818 pupils wrote the National Senior Certificate Examination for 2011 colloquially known as the matric in South Africa [4]. Of those pupils who wrote the matric examinations, only 224635 pupils wrote the mathematics examination. Of those 224635 who wrote the mathematics examination, only 104033 passed the examination. And to qualify as a pass, the pupil needed to only achieve $30 \%$ on the examination [4].

Whether one considers those 104033 successful pupils as a percentage of the number of pupils who wrote the mathematics examination $(46 \%)$; or whether one considers those 104033 successful pupils as a percentage of the number of matriculants $(17 \%)$; or whether one considers those 104033 successful pupils as a percentage of the total number of pupils who had started school 12 years prior $(9.6 \%)$, one can only conclude that there is space for initiatives that can help with mathematics education in South Africa.

Dr Math is one such initiative. Dr Math is also an example of a virtual volunteer project. It uses a virtual volunteer network of mathematics tutors who help primary and secondary school pupils. The school pupils use chat facilities on their cell phones and can receive immediate help with their homework. The volunteer tutors use normal Internet-based workstations [5].

\section{ClarifiCATION OF TERMS}

Scientists and researchers in different fields often use the same word with dramatically different definitions. This needs to be clarified before confusion mounts.

Computer scientists use the word synchronous when describing computer communication protocols where 
responses must be sent in a timely manner. A computer scientist would say that HTTP (HyperText Transfer Protocol) is a synchronous protocol. When a computer (such as a personal computer or cell phone) makes an HTTP call to a web server, the receiving web server must reply back immediately to the originating computer with the requested web page in a timely manner. However, when a computer scientist considers a chat protocol such as XMPP (eXtensible Messaging and Presence Protocol), he or she would classify it as an asynchronous protocol. When one computer (or cell phone) sends an XMPP message to another computer (or cell phone), the receiving device is not compelled to reply.

Linguists (and social scientists), on the other hand, view the world through the eyes of the human and, as such, would consider XMPP to be a synchronous protocol in the eyes of the human. When a person (as opposed to a computer) sends an XMPP message to another person, the second person is socially obligated to reply immediately. XMPP can be considered to be a synchronous or near-synchronous communication medium [6]. With reference to Tagliamonte's [7] description of online chatting, the XMPP protocol is synchronous because it supports two human beings communicating with each other in a reciprocal (turntaking) manner.

In this paper, the terms asynchronous and synchronous are used to describe human-to-human communication and not computer-to-computer communication.

\section{Virtual VolunteEring PROJECTS}

There are a number of very successful virtual volunteering projects.

Project Gutenberg did not necessarily start as a virtual volunteering project. In 1971, Michael Hart started Project Gutenberg with the goal being to electronically make available free literary works belonging in the public domain. During the first years of the project, Hart himself typed in the books. In the mid-1990s, however, Hart started to coordinate the work of dozens and then hundreds of volunteers throughout the world [8].

Virtual volunteers to Project Gutenberg compile a digital library of books which have expired copyrights. Some of the volunteers type in the text of the books. Some of the volunteers proofread books [9]. Other volunteers are needed to search for eligible books, create CDs and DVDs for people who do not have access to the Internet, promote the project, etc [10].

According to Project Gutenberg's website, as of April 2012, there are over 38,000 free ebooks hosted on the website [10].

Project Librivox is another example of a project working with a network of virtual volunteers. Project Librivox was started in 2005 by Hugh McGuire with the objective of making all books in the public domain free in an audio format on the Internet. Librivox welcomes volunteers from around the world to record books, plays, and poetry [11].

Although both projects are extremely successful virtual volunteer projects, the volunteers need to donate a substantial amount of time. For example, a volunteer needs to read an entire chapter of a book or proofread an entire chapter of a book.

It would not be possible to donate ten minutes of one's time to either project. However, there are virtual volunteering projects where it is possible to donate small amounts of time.

Micro-volunteering allows people to volunteer very short periods of time. There have been a number of microvolunteering projects. Many of these are asynchronous requests for small pieces of work. For example, the website Mobile Volunteering [12] seeks micro-volunteers to tag and photograph places where trees are needed, survey science careers, translate small pieces of text, and donate photographs to charity. The website Volunteer Match [13] seeks micro-volunteers to assist in securing funding, updating charity websites, capturing information into a database, and planning events.

Whilst these requests may be important and may only require small amounts of time, there is no sense of timely urgency that they must be completed within the next five to ten minutes.

\section{DR MATH}

Dr Math is a virtual volunteer project based in South Africa which allows primary and secondary school pupils to use text based chat facilities on their cell phones to reach volunteer tutors to help them with their mathematics homework in a timely manner[5]. The volunteer tutors use traditional Internet based workstations and the school pupils use their cell phones. The platform which links the tutors and the pupils together hides all personal identities and keeps all parties anonymous.

The Dr Math project was started in 2007. At that point in time the researchers were unsure of whether or not school pupils, especially teenagers, would even want to chat about mathematics on their cell phones. There was a fear by the researchers that teenagers might feel that mathematics would contaminate their cell phones. Unexpectedly thousands of pupils started asking for help with their mathematics homework and the project quickly outgrew the research framework which had originally been developed. The platform was completely redeveloped in 2010 to cater for scalability issues. This new platform, $\mathrm{C}^{3} \mathrm{TO}$ (Chatter Call Centre/Tutoring Online) was specifically designed to handle the scalability issues which had arisen [14].

A typical session by a volunteer tutor involves the tutor chatting with twenty to thirty pupils concurrently using a web interface as can be seen in Illustration 1. From the pupils' points of view, however, each pupil is having a oneto-one conversation with a tutor. If more than one tutor is logged in and tutoring, $\mathrm{C}^{3} \mathrm{TO}$ attempts to determine which of the tutors are the least busy (by monitoring response times and message lengths) and when a new pupil poses a question, $\mathrm{C}^{3} \mathrm{TO}$ forwards the question to the least busy tutor [15]. 


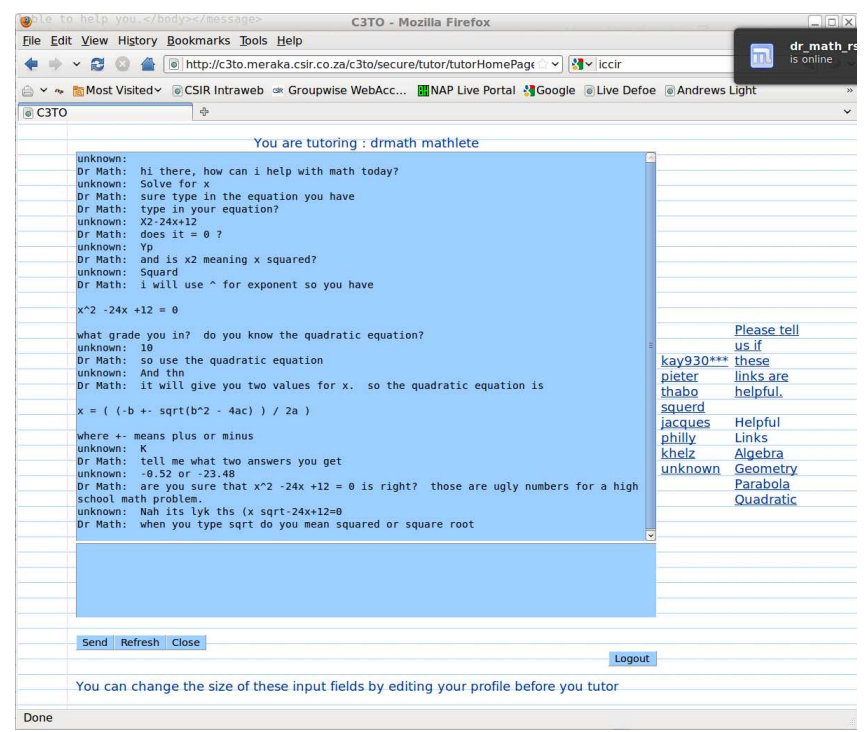

Illustration 1: Typical web-based tutoring session

A typical conversation between a tutor and a pupil consists of approximately ten to twenty messages sent between the two participants. The messages from the pupils are typically conducted in "SMS lingo" or "Net lingo" where vowels are optional and where symbols and numerals are used in the place of some syllables and words. For example:

pupil: ey

tutor: hi there can i help with math today

pupil: how do i factorize

tutor: numbers or polynomials

pupil: polynoms

tutor: $\mathrm{i}$ will use ${ }^{\wedge}$ for exponet. like this $\mathrm{x}^{\wedge} 2+6 \mathrm{x}+8$

pupil: ok

tutor: so now you need to find two ints which add

tog to make 6 but multiply togeteher to make 8 . what are those two numbers

pupil: 2.4

tutor: yes so the factors are $(x+2)$ and $(x+4)$ what about this one $\mathrm{x}^{\wedge} 2+8 \mathrm{x}+15$

pupil: 4 . an 3

tutor: what is 4 times 3 ?

pupil: so it must mltply 215 ?

tutor: yes

In the existing configuration, however, tutors need to be online for a minimum of approximately an hour at a time. There are a number of reasons for this. Firstly, although there are published schedules of when tutors are available, it takes fifteen to twenty minutes for the school pupils to really start asking questions once they are sure that a tutor is available. Secondly, once a tutor is chatting to pupils, it is socially difficult for the tutor to just stop chatting. As mentioned previously, the tutor is often chatting to twenty pupils and the tutor needs to actually wind up those conversations politely. Unfortunately, while the tutor is attempting to wind up the existing conversations, new pupils start new conversations.

The Dr Math tutors, however, often become overwhelmed with questions. South Africa is a country with standardized school examination times and, in some cases, standardized school examinations. The academic year runs from the middle of January until the end of November. Around the country, June is reserved for mid-year examinations. For Grade 12's, August is reserved for preliminary school leaving examinations. And mid-October through end November is reserved for end-year examinations. During the run-up to these examination periods, there are times when tutors are attempting to chat with up to fifty pupils at one time. Alas, in such situations, the tutor can not give good responses to all the pupils.

Statistical analysis of the historical conversations which occurred between January 1, 2012, and October 1, 2012, found that the average length of time for a conversation between a tutor and pupil was approximately 15 minutes and the median length of time was between 7 and 8 minutes. Illustration 2 shows the distribution of conversation lengths with the majority of conversations being less than 10 minutes.

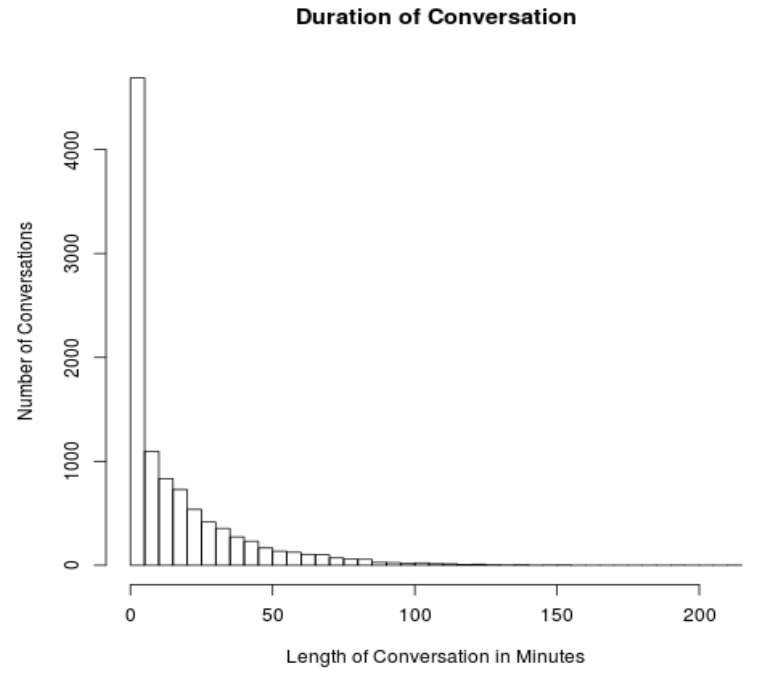

\section{Illustration 2: Histogram of lengths of conversations measured in minutes}

In such cases, a mechanism needed to be developed to allow additional tutors to volunteer for extremely short periods of time possibly as short as ten minutes. In addition, if these tutors could be sourced while they were away from the workstations and allowed to tutor using their cell phones, then the pupil load during these peak periods could be satisfied.

This paper describes the work done towards a synchronous micro-volunteering platform where volunteers 
can tutor for extremely short periods of time using the convenience of their cell phone when demand requires.

\section{EXISTING $\mathrm{C}^{3}$ TO PLATFORM}

The Dr Math service is hosted on an instance of the $\mathrm{C}^{3} \mathrm{TO}$ platform. $\mathrm{C}^{3} \mathrm{TO}$ is a JEE (Java Enterprise Edition) application. $\mathrm{C}^{3} \mathrm{TO}$ consists of two major portions which are connected via a common database. The school pupils access $\mathrm{C}^{3} \mathrm{TO}$ through chat protocols on their cell phone. This is handled by the mobile portion of the $\mathrm{C}^{3} \mathrm{TO}$ architecture which is based on the Mobicents communication platform. The volunteer tutors access $\mathrm{C}^{3} \mathrm{TO}$ through a normal Internet website. That web application uses JbossSeam, JSF (Java Server Faces), and Postgresql. AJAX (Asynchronous JavaScript and XML) is used by the JSF pages to push the pupils' questions to the tutors [14].

The school pupils are typically minor children (under the age of 18 years). Their contact details must be kept hidden from all participants and the platform, therefore, hides their identities.

Illustration 3 (in black) shows the basic architecture. Two chat protocols are supported for access by the school pupils. The pupils can use either XMPP or MXit. While XMPP is a international standard, MXit is a mobile text based chat protocol which was developed in South Africa and boasts millions of users in South Africa [16, 17]. Although the intricacies of the two protocols are different, they have very broad similarities. Both protocols require that users load special software onto their cell phones to communicate with a server, are session based, allow users to create a roster of friends or contacts with whom they wish to communicate, and also allow the central server to push messages to the user.

The existing tutor access to $\mathrm{C}^{3} \mathrm{TO}$ is through a traditional webpage using HTTP. The sessions are maintained by JbossSeam. AJAX is used to simulate the push of messages from the pupils to the tutors. This existing web access was not suitable for cell phone access or push access because of two reasons: 1) AJAX did not work on all cell phone browsers 2) A simulated push of messages using AJAX was not an efficient way to handle mobile communication.

$\mathrm{C}^{3} \mathrm{TO}$ provides timely synchronous (in the eyes of the human participants) communication between pupils and tutors. The pupils need immediate help with their mathematics homework and this architecture provides that within the limitations of the human tutors.

The existing $\mathrm{C}^{3} \mathrm{TO}$, however, needed to be modified and enhanced to cater for micro-volunteering.

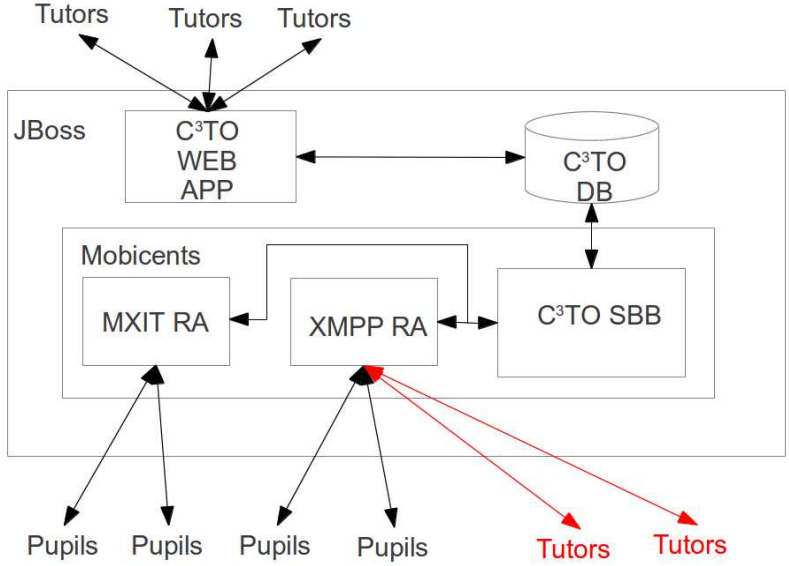

\section{Illustration 3: Pre-existing architecture (in black) and modifications (in red)}

\section{ETHICS AND SAFETY OF MiNOR CHILDREN}

Dr Math is a project which links minor children (under the age of 18 years) to virtual volunteers without obtaining parental permissions for this contact. The Dr Math project did receive an ethics clearance. It is a requirement of this clearance that all identities are hidden from all parties. This requirement precluded just posting the chat addresses of tutors on an open webpage. When the minor children and the tutors interact, their identities and contact details must be hidden from each other.

Any modifications which would need to be done to the $\mathrm{C}^{3} \mathrm{TO}$ platform would have to honour the requirements of the ethics clearance.

\section{MOdifications to $\mathrm{C}^{3} \mathrm{TO}$}

The $\mathrm{C}^{3} \mathrm{TO}$ platform was modified to allow communication with the volunteer tutors using XMPP in addition to the normal web interface as can be seen in Illustration 3 (in red). When volunteers registered to become a tutor, besides giving normal contact information such as name, email address, and cell phone number, the tutor was also asked if he or she wished to tutor via chat facilities and also whether or not he or she could be contacted during peak periods via chat facilities for unscheduled peak period tutoring.

During the course of normal operation, when the $\mathrm{C}^{3} \mathrm{TO}$ platform determined that the delay in attending to pupils' questions was unacceptably high (this was a configuration option), XMPP messages were sent to volunteer tutors who were not logged in but indicated that they could help during peak periods were sent out.

These tutors could then use XMPP on their cell phones and send a specific message to one of a handful of chat addresses which were monitored by the $\mathrm{C}^{3} \mathrm{TO}$ platform. 
Because of the physical limitations of tutoring mathematics on a cell phone, the tutor would only have one tutoring session live per chat address. However, $\mathrm{C}^{3} \mathrm{TO}$ did support the facility where the tutor could contact a fixed number of different chat addresses thus being allowed to tutor any number of pupils up to that fixed number.

\section{TESTING ENVIRONMENT}

The enhancements to $\mathrm{C}^{3} \mathrm{TO}$ to cater for synchronous micro-volunteering were tested using Android phones only.

The algorithm which assigned school pupils to tutors was modified in the following manner:

1. If a question was asked by a pupil and no tutors were logged in, then alerts were sent to potential volunteer tutors via XMPP (depending on the tutors preference) to see if they could help.

2. If a question was asked by a pupil and a tutor was logged in and the tutor was extremely busy (with the definition of busy being configurable), then alerts were sent to potential tutors for help.

3. If a question was asked by a pupil and a tutor was logged in but the tutor was not extremely busy, then the pupil was assigned to the existing tutor and no alerts were sent out.

\section{Results}

In this investigation, we learned lessons at two levels; firstly, whether or not synchronous micro-volunteering can work at all and, secondly, whether this form of microvolunteering can work for mathematics tutoring.

In the first case, existing tutors for Dr Math were eager to assist pupils with their mathematics homework for short periods of time. In the case of the Dr Math project, this synchronous micro-volunteering facility was used at times of peak load where there were many pupils asking more questions than the existing tutors could handle. This is particularly true during the run up to nationally set school leaving examinations in South Africa know as the matric.

In the second case, micro-volunteering on Android phones for the specific reason of tutoring mathematics had a drawback of handling a mixture of text symbols along with the mathematics symbols such as $+{ }_{-}^{*}$ and /. On many touch phones the letters and the symbols were on two different virtual keyboards and typing in a message such as

$$
\mathrm{x}^{\wedge} 2+7 \mathrm{x}+12
$$

required a high number of inconvenient swapping back and forth between keyboards. This slowed down the response time of the tutor. In the particular case of the Dr Math project, the tutors felt it was very difficult to use and only used the micro-volunteering option a couple of times before returning to the traditional web-based tutoring interface.

The researchers are satisfied that concept of microvolunteering works, the instantiation of a synchronous micro-volunteering facility in $\mathrm{C}^{3} \mathrm{TO}$ works and can be used to alleviate peak demand periods. However, in this particular case, the use of micro-volunteering on cell phones was, perhaps, not particularly suitable for mathematics education because of the mixture of letters and symbols used in the messages sent by the tutors.

Future research in this area could include the creation of a custom Android application to specifically cater for mathematics tutoring via XMPP. In such a future application, the default keyboard could include letters, digits, and common mathematical symbols such as $+{ }_{-} *$ and $/$.

\section{CONCLUSION}

A wide variety of organizations harness the power of volunteers. Many organizations look for physical volunteers such as the Red Cross and the Scouting movement. Some organizations look for virtual volunteers such as the Gutenberg Project and Librivox Project.

Dr Math is a virtual volunteering project where people can volunteer to help school pupils with their mathematics home using traditional Internet-based workstations.

This paper described an enhancement to the Dr Math project which allowed synchronous micro-volunteering. Micro-volunteering allows potential volunteers to donate their time in small packets of ten minutes. The Dr Math project implemented this micro-volunteering facility using chat protocols on cell phones allowing these microvolunteers to be on-the-move. This allowed people to volunteer their time and expertise while they were waiting in a queue for a bus or at the doctor's office.

\section{ACKNOWLEDGEMENT}

L.B. thanks the Rupert Family Trust for the Rupert Gesinstigting Award received in 2011. R.B.'s work is partially funded by the National Research Foundation of South Africa.

\section{REFERENCES}

[1] J. Wilson and M. Musick. Who cares? toward an integrated theory of volunteer work. Am. Sociol. Rev. 62(5), pp. 694-713. 1997.

[2] J. Cravens. Virtual volunteering: Online volunteers providing assistance to human service agencies. Journal of Technology in Human Services 17(2), pp. 119-136. 2000.

[3] Department of Education, "Trends in education macro-indicators: South africa," Department of Education, Pretoria, 2009.

[4] Department of Basic Education, "Report on the national senior certificate examination 2011," Department of Basic Education, Pretoria, 2011.

[5] A. Botha and L. Butgereit, "Dr Math: A Mobiled Scaffolding Environment," International Journal of Mobile and Blended Learning, vol. 4, pp. 15-29, 2012.

[6] J. S. Durham. Topic detection in online chat. Topic Detection in Online Chat 2009.

[7] S. A. Tagliamonte and D. Denis. Linguistic ruin? LOL! instant messaging and teen language. American Speech 83(1), pp. 3-34. 2008.

[8] M. Lebert. Project Gutenberg (1971-2008) 2008. 
[9] B. Stroube. Literary freedom: Project gutenberg. Crossroads 10(1), pp. 3. 2003. 18 ,

[10] Gutenberg, "Project Gutenberg," vol. 2010, October

[11] R. Van Horn. Online books and audiobooks. Phi Delta Kappan 89(2), pp. 154-155. 2007.

[12] Anonymous "Mobile Volunteering," vol. 2013, .

[13] Anonymous "Volunteer Match," vol. 2013, 2013.

[14] L. Butgereit, "C ${ }^{3} \mathrm{TO}$ : a Scalable Architecture for Mobile Chat Based Tutoring," 2011.

[15] L. L. Butgereit and R. A. Botha. A busyness model for assigning tutors to pupils in a mobile, online tutoring system: A look at C 3 TO. Presented at Proceedings of the 2010 Annual Research Conference of the South African Institute of Computer Scientists and Information Technologists. 2010, .

[16] W. Chigona and A. Chigona. MXit up in the media: Media discourse analysis on a mobile instant messanging system. The Southern African Journal of Information and Communication 9(0), pp. 42-57. 2009.

[17] W. Chigona, A. Chigona, B. Ngqokelela and S. Mpofu. MXIT: Uses, perceptions and self-justifications. Journal of Information, Information Technology, and Organizations 4pp. 1-16. 2009. 